\title{
Daylight Spectrum Index: Development of a New Metric to Determine the Color Rendering of Light Sources
}

\author{
Ignacio Acosta
}

\begin{abstract}
Nowadays, there are many metrics to determine the color rendering provided by a light source. The Color Rendering Index is the most widely metric used to determine the accuracy of a light source, according to eight color samples. However, this metric does not allow a proper measurement for LED lamps. Recently, the Color Quality Scale was defined, extending the color samples selected by previous metrics in order to improve the analysis of LED lamps. However, the results of both metrics for a low correlated color temperature use as reference the Standard Illuminant $A$, that is to say, the color rendering of an incandescent lamp, which does not represent the real conditions of the natural color rendering. Currently, new metrics appears, such as the TM-30-15, based on 99 color samples and a complex calculation. According to this context, a new metric is proposed, based in the Spectral Power Distribution of daylighting. The daylight spectrum is corrected by the photopic luminosity function, as well as the spectrum of the light source sample. Both corrected spectrums are compared in order to determine the Daylight Spectrum Index. This new metric represents a better definition of the LED lamps for a high correlated color temperature, as can be deduced from the analyzed surveys.
\end{abstract}

Index Terms-Daylight, spectrum, index, color rendering, color temperature, color quality scale.

\section{INTRODUCTION}

Currently, the Color Rendering Index (CRI) is the only metric recognized by the Commission Internationale de l'Eclairage in order to determine the color rendering properties of a light source [1]. This metric is based in the analysis of eight non-saturated color samples, close to white. The selected samples are located in the CIE color space 1960 and the color deviation produced by the light source is compared with that observed for the light reference. However, the CRI has shown many deficiencies to determine the color rendering of a light source [2]-[4] mainly produced by the following statements:

- The CIE color space 1964 is obsolete: According to the Mc Adams ellipses [5], which determine the areas with equal color perception in the CIE color space, the color distribution of the current space to determine the CRI does not correspond to the human perception.

- The color samples are not enough to evaluate the color rendering: Following the CRI procedure [1], eight low

Manuscript received November 5, 2016; revised March 12, 2017. This work was supported in part by the University of Seville.

Ignacio Acosta is with the Instituto Universitario de Arquitectura y Ciencias de la Construcción, Sevilla. 2 Reina Mercedes Ave. Seville, Spain (e-mail: iacosta@us.es). saturated color samples do not represent the full color spectrum of a light source [6].

- The calculation of the CRI for a light source depends on the mean deviation of each color sample [1], therefore, the lower performances are unnoted in the overall value.

- The light source reference for the CRI calculation varies according to the correlated color temperature [1], hence there is no a reliable and sole reference to calculated the performance of different light sources.

According to the previous statements, the CRI offers a poor estimation of light-emitting diodes (LED) [7], hence the CIE has promoted to revise this metric [8]. In order to improve the results for LED lamps, the Color Quality Scale (CQS) was defined [9], establishing the following improvements:

- 15 new color samples: according to saturated colors.

- An update of the color space: for the CQS calculation, the CIE color lab is used in the place of the CIE color space 1964.

- The chromatic adaptation is updated to CMCCAT2000.

- The deviation is calculated according to the Root Mean Square (RMS), hence the lower performances in specific color samples are noted in the overall result.

However, despite the improvements of this new metric, the light source references to contrast the color rendering still depends on an incandescent lamp [10], which does not represent the real conditions of the natural color rendering.

Due to the inaccuracy of the proposed metrics, new definitions of the color rendering index appears, such as the CRI2012 [11] and the CRI-CAM02UCS [12]. One of the most current metrics corresponds to the TM-30-15, developed by the IESNA [13], which analyzes 99 color samples in order to determine the higher accuracy in the color rendering of a light source by means of a complex calculation.

As seen in this introduction chapter, it is worth noting that there is a constant updating of the metrics for colorimetry, because there is not a solid criteria to establish the color performance of a light source.

In addition, most of the new metrics that attempt to determine the color performance of a light source are based in a color chart, which always depends on a subjective interpretation of the color distribution. However, the color rendering can also be determined analyzing the Spectral Power Distribution, which describes the power per unit wavelength of a light source.

Moreover, it can be deduced that daylighting should be the sole light source reference to determine the color rendering, although most of the proposed metrics are also based on the spectrum distribution of an incandescent lamp. 


\section{OBJECTIVES}

The aim of this research is to determine a new metric which serves to define the color rendering of a light source, considering the following statements:

- The new metric must be based in a sole light source reference, unlike other metrics described before. This reference must correspond to daylighting.

- The new metric must be argued in the spectral power distribution of the light sources, avoiding any measurement on a color chart, which depends on a subjective color interpretation. Therefore, there is no any color sample to determine the color rendering.

- The new metric should not depend on any chromatic adaptation, which can provide a correction of the color rendering according to psychological conditions, but also includes a subjective interpretation of the human perception of colors.

- The new metric should consider the environmental conditions to determine the proper color perception according to the location and typical weather conditions.

Following the above statements, the Daylight Spectrum Index is defined.

\section{METRIC DEFINITION}

The Daylight Spectrum Index (DSI) is defined as the ratio of the area defined by the photopic perception of the Spectral Power Distribution of the studied light source and that defined by daylight. In other words, the DSI represents the affinity of the light source to the daylight perception, according to its Spectral Power Distribution (SPD).

Due to DSI is calculated using the contrast of the Spectral Power Distributions, there are no color sample, color temperature or color chart which affect to the results obtained. Therefore, this metric does not depend on any color space chart or chromatic adaptation. The DSI can be assessed according to different ranges: blue, green and red, comparing the areas contained between two wavelengths.

Below it is shown the calculation process of the Daylight Spectrum Index:

\section{A. Definition of the Sky Spectrum}

The sky spectrum must be defined, according to the typical weather conditions where the performance of the light source is conducted.

The standard sky conditions for calculating the Daylight Spectrum Index corresponds to the CIE D65 [14], which represents the most common daylight spectrum for Northern Europe. However, the daylight spectrum must change according to the typical weather conditions of the location where the studied is carried-out. Considering a mainly overcast sky throughout the year, as in the case of Northern England or the Scandinavian countries, an overcast sky spectrum must be used as reference to determine the color rendering of a light source. Moreover, for those countries located in the Mediterranean climate, a clear sky spectrum must be applied.

The proper choice of the daylight spectrum is key to take into account the environmental conditions where the subject is assessing the affinity of the light source to the daylight reference. Therefore, the sky spectrums proposed according to different locations in Europe are described below and shown in Fig. 1:

- CIE D50: Overcast sky distribution: England, Scotland, Ireland, Scandinavian countries, Denmark.

- CIE D65: Average sky distribution for Northern Europe: Northern France, Germany, Switzerland, Austria, Poland.

- CIE D75: Mainly clear sky distribution: Southern France, Northern Spain, Northern Italy.

- CIE D90: Clear sky distribution: Southern Spain, Southern Italy, Greece, Romania.

\section{Daylight Spectrum Power Distributions}

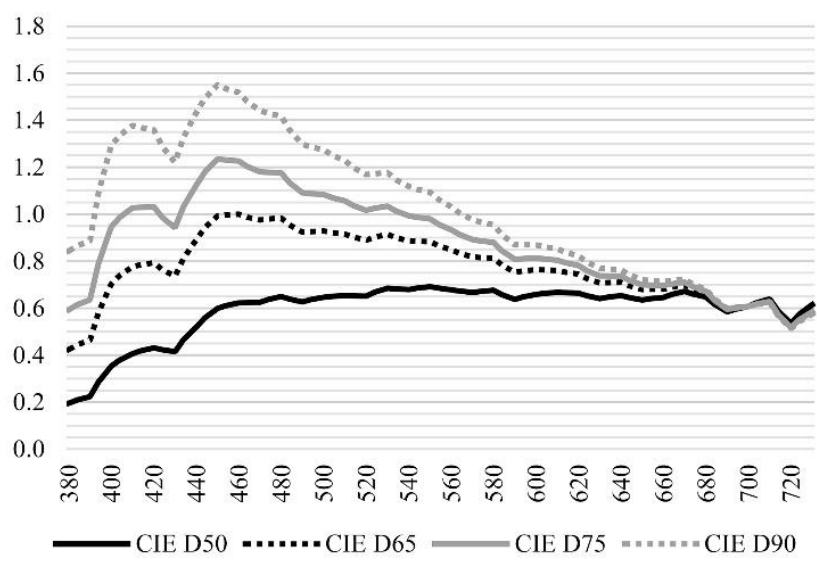

Fig. 1. Daylight spectrum power distributions of the most common skies for Europe.

It is worth noting that this proposal is based on the statistical sky conditions of each location, but it only serves as a recommendation. The appropriate SPD should be chosen according to the average daylight spectrum throughout the year in the selected location.

\section{B. Definition of the Photopic Perception of the Sky SPD}

After choosing the Spectral Power Distribution of the sky reference, the photopic perception must be applied. The photopic sensitivity function describes the average spectral sensitivity of human visual perception of brightness. The CIE luminosity function $\mathrm{V}(\lambda)$ is a standard function established by the CIE [15] (Fig. 2):

Photopic sensitivity function

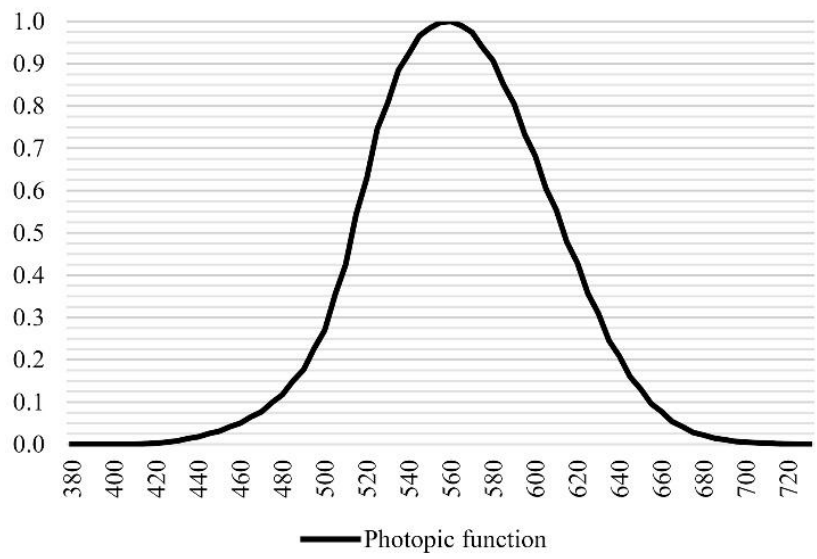

Fig. 2. Photopic sensitivity function, also known as function $\mathrm{V}(\lambda)$.

The Spectral Power Distribution of the sky can be 
modified according to the human perception defined by the photopic function, as follows:

$$
f(\lambda)=V(\lambda) \cdot S K Y(\lambda)
$$

where $V(\lambda)$ corresponds to the photopic sensitivity function and $\operatorname{SKY}(\lambda)$ is equal to the Spectral Power Distribution of the sky, both as a function of the wavelength.

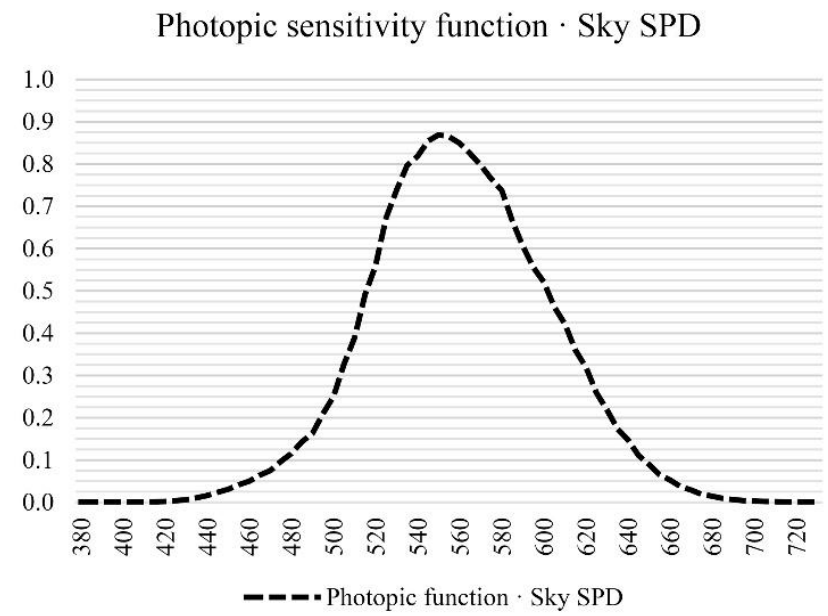

Fig. 3. Spectral Power Distribution of the sky reference modified by the photopic sensitivity function.

Fig. 3 shows the Spectral Power Distribution of the daylight, perceived by the human being. It serves as a reference for the perception of other light sources.

\section{Normalization of the Resulting Sky SPD}

After defining the human perception of the daylight, the resulting function must be normalized. The normalization of a function must be carried out as follows:

- Calculate the specific total perceived power (Ts) by integrating the area under the resultant curve.

- Calculate a scalar $(\mathrm{P})$ using the following function: $\mathrm{P}=\mathrm{Ta} / \mathrm{Ts}$

Where Ta represents an arbitrary value used to scale the results. If a specific scale range if not required then just use a Ta value of 1 .

- Multiply the value of each point in the original data set by the scalar $(\mathrm{P})$ to produce a normalized data set.

The previous process can be express as follows:

$$
f_{N}(\lambda)=f(\lambda) \frac{T_{a}}{\int_{380}^{730} f(\lambda) d \lambda}
$$

where $f(\lambda)$ corresponds to the modified SPD of the sky, as deduced from equation (1) and Ta is the arbitrary value used to scale the results.

This process serves to establish a common total perceived power for the daylight reference and also for the analyzed light source, in order to properly compare both light spectrums.

Fig. 4 shows the modified SPD of the sky, according to the human perception defined by the photopic function, and normalized following a scalar value of 10 . The same value must be used for the normalization of the analyzed light source.

\section{Photopic Perception of the Studied Light Source}

As in the previous case, the photopic perception of the studied light source is defined, according to the function $V(\lambda)$, expressed as follows:

\section{Photopic sensitivity function $\cdot$ Sky SPD Normalized}

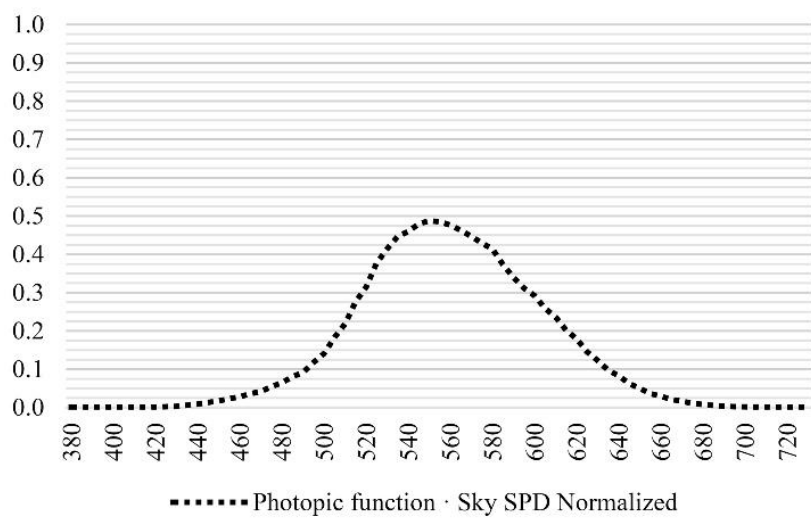

Fig. 4. Spectral Power Distribution of the sky reference modified by the photopic sensitivity function and Normalized for $\mathrm{Ta}=10$.

$$
\delta(\lambda)=V(\lambda) \cdot \operatorname{LIGHT}(\lambda)
$$

where $V(\lambda)$ corresponds to the photopic sensitivity function and $\operatorname{LIGHT}(\lambda)$ is equal to the Spectral Power Distribution of the studied light source, both as a function of the wavelength.

The photopic perception of a LED lamp with a correlated color temperature (CCT) of $6,500{ }^{\circ} \mathrm{K}$ is shown in Fig. 5.

\section{Photopic sensitivity function $\cdot$ Light source} SPD

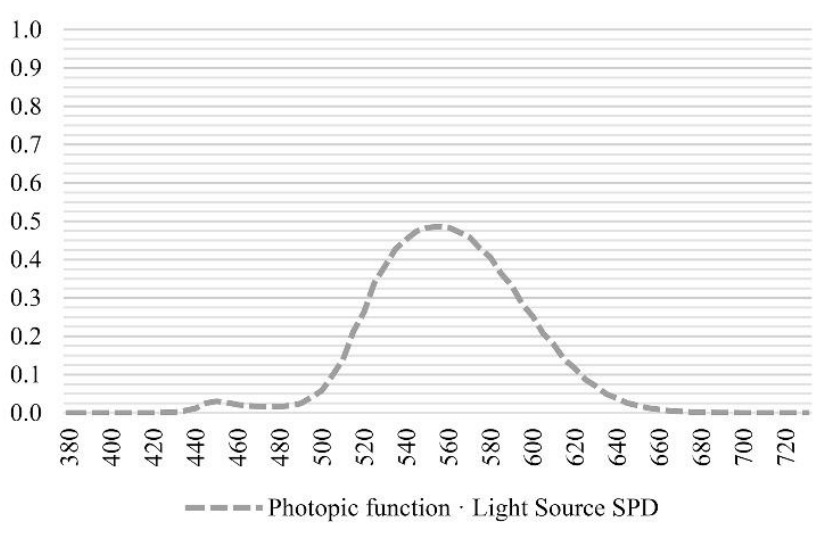

Fig. 5. Spectral Power Distribution of the studied light source modified by the photopic sensitivity function.

\section{E. Normalization of the Resulting Studied Light Source}

Following the method described form the daylight reference, the resulting SPD of the light source is normalized, according to equation (4):

$$
\delta_{N}(\lambda)=\delta(\lambda) \frac{T_{a}}{\int_{380}^{730} \delta(\lambda) d \lambda}
$$

Fig. 6 shows the modified SPD of the studied LED lamp, according to the photopic function and normalized for a scalar value of 10 .

\section{F. Ratio of the Resulting Areas}

Finally, the Daylight Spectrum Index (DSI) is defined as 
the ratio of the area defined by the photopic perception of the Spectral Power Distribution of the studied light source and that defined by daylight, that is to say:

Photopic sensitivity function · Light source SPD Normalized

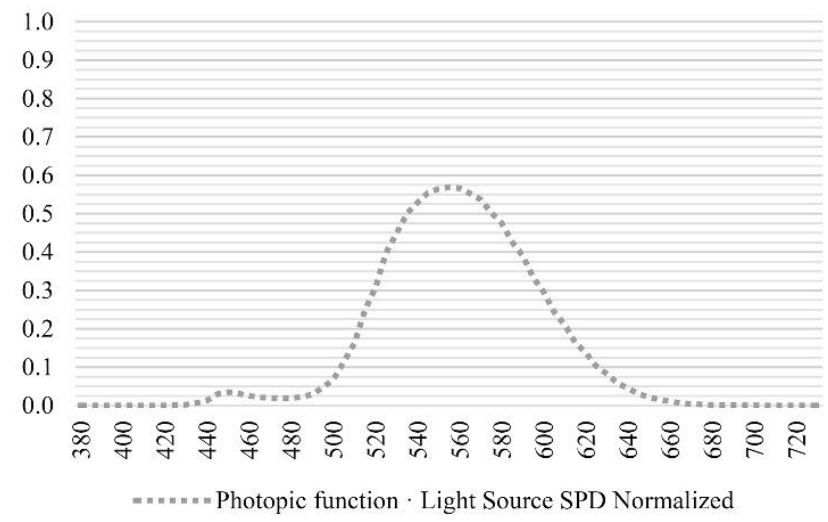

Fig. 6. Spectral power distribution of the light source modified by the photopic sensitivity function and Normalized for Ta $=10$.

$$
\operatorname{DSI}(\%)=100 \cdot \frac{\int_{380}^{730} \operatorname{Min}\left(\delta_{N}(\lambda), f_{N}(\lambda)\right) d \lambda}{\int_{380}^{730} f_{N}(\lambda) d \lambda}
$$

where $\delta_{N}(\lambda)$ is the normalized function of the perceived light source according to the photopic vision and $\mathrm{f}_{\mathrm{N}}(\lambda)$ is the same function for the daylight reference.

\section{G. Definition of the Metric According Different Hues}

The DSI can be defined according to different hues of the visible spectrum, setting the wavelength interval to define the SPD ratios, as seen in equations (6), (7) and (8):

$$
\begin{gathered}
\operatorname{DSI}_{B L U E}(\%)=100 \cdot \frac{\int_{380}^{530} \operatorname{Min}\left(\delta_{N}(\lambda), f_{N}(\lambda)\right) d \lambda}{\int_{380}^{530} f_{N}(\lambda) d \lambda} \\
\operatorname{DSI}_{\text {GREEN }}(\%)=100 \cdot \frac{\int_{530}^{590} \operatorname{Min}\left(\delta_{N}(\lambda), f_{N}(\lambda)\right) d \lambda}{\int_{530}^{590} f_{N}(\lambda) d \lambda} \\
\operatorname{DSI}_{R E D}(\%)=100 \cdot \frac{\int_{590}^{730} \operatorname{Min}\left(\delta_{N}(\lambda), f_{N}(\lambda)\right) d \lambda}{\int_{590}^{730} f_{N}(\lambda) d \lambda}
\end{gathered}
$$

Fig. 7 shows the Daylight Spectrum Index for the studied LED lamp with a CCT of $6,500^{\circ} \mathrm{K}$, compared with a daylight spectrum CIE D65, obtaining an overall DSI equal to $90.09 \%$, a DSI BLUE $_{\text {of } 73.90 \%}$, a DSI $_{\text {GREEN }}$ of $100.00 \%$ and a DSI $I_{\text {RED }}$ of $84.74 \%$.

\section{METRIC EXAMPLES}

After defining the Daylight Spectrum Index, several examples are shown in order to demonstrate the potential of this new metric.

Fig. 8 shows the Daylight Spectrum Index for an incandescent lamp with a CCT of $2,812^{\circ} \mathrm{K}$, compared with a daylight spectrum CIE D65, obtaining an overall DSI equal to $84.48 \%$, a DSI $\mathrm{BLUE}_{\mathrm{B}}$ of $54.83 \%$, a DSI $\mathrm{BREEN}_{\mathrm{G}}$ of $87.97 \%$ and a DSI $_{\text {RED }}$ of $100.00 \%$.
Daylight Spectrum Index calculation

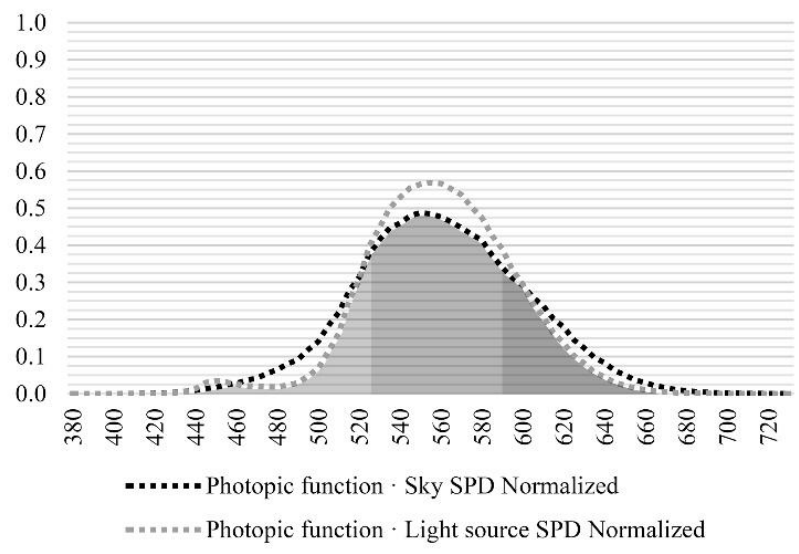

Fig. 7. Daylight spectrum index for a LED lamp with CCT $6,500{ }^{\circ} \mathrm{K}$ compared with a daylight spectrum CIE D65.

Daylight Spectrum Index calculation

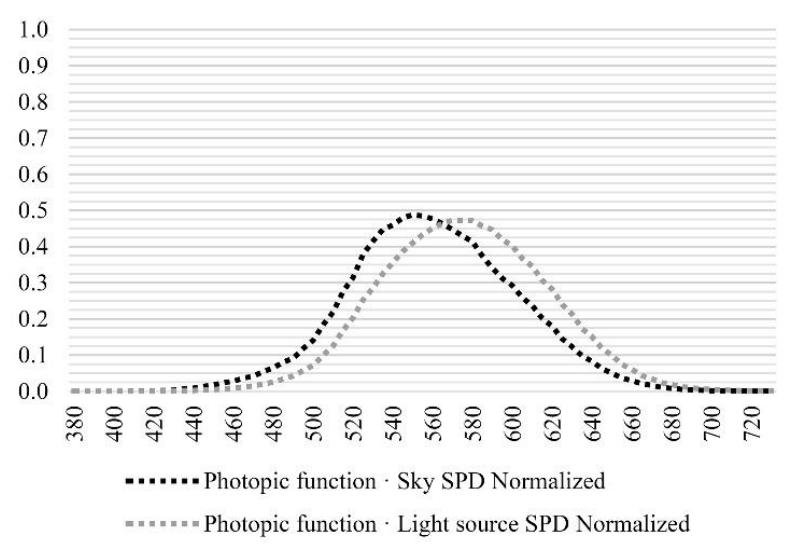

Fig. 8. Daylight spectrum index for an incandescent lamp with CCT 2,812 ${ }^{\circ} \mathrm{K}$ compared with a daylight spectrum CIE D65.

Daylight Spectrum Index calculation

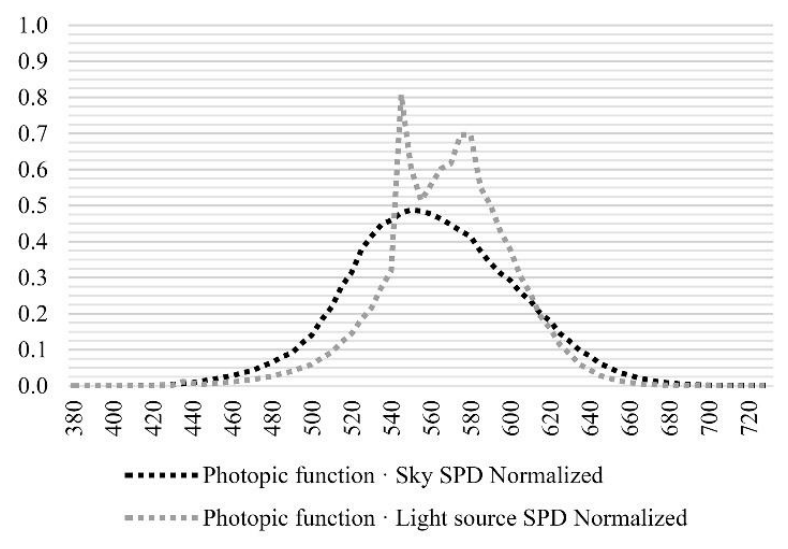

Fig. 9. Daylight spectrum index for a fluorescent F34 Cool white lamp with CCT $4,070^{\circ} \mathrm{K}$ compared with a daylight spectrum CIE D65.

Fig. 9 shows the Daylight Spectrum Index for a fluorescent F34 Cool white lamp with a CCT of $4,070^{\circ} \mathrm{K}$, compared with a daylight spectrum CIE D65, obtaining an overall DSI equal

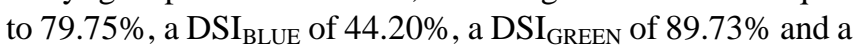
DSI $_{\text {RED }}$ of $88.34 \%$.

As deduced from the previous examples, the LED lamp produces a better perception of the blue hues and a slightly higher rendering of the overall colors.

Moreover, the incandescent lamp allows a better rendering of the red hues, achieving a $100 \%$ in such wavelength 
interval.

\section{EXPERIMENTAL}

12 specialists in painting sculpture were asked about their preferences in color rendering. The specialists analyzed two different lamps:

- LED lamp with a CCT 6,500 ${ }^{\circ} \mathrm{K}, 600 \mathrm{~lm}$, CRI 72.00\%, CQS $73.00 \%$.

- Incandescent lamp with a CCT 2,812 ${ }^{\circ} \mathrm{K}, 600 \mathrm{~lm}, \mathrm{CRI}$ $100.00 \%$, CQS $98.00 \%$.

In the case of the LED lamp, the overall perception of the 12 specialists is shown in Fig. 10.

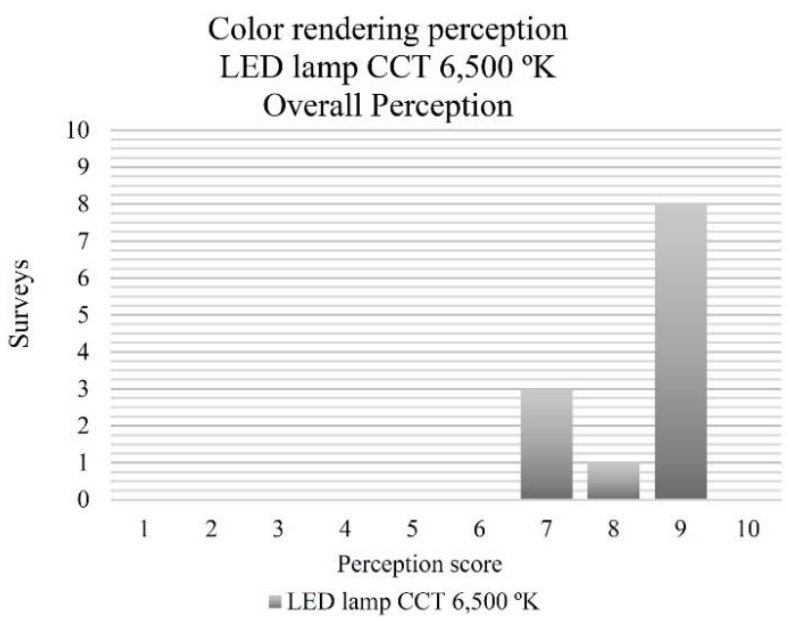

Fig.10. Overall color rendering perception for a LED lamp with CCT $6,500{ }^{\circ} \mathrm{K}$.

Color rendering perception

Incandescent lamp CCT $2,812^{\circ} \mathrm{K}$

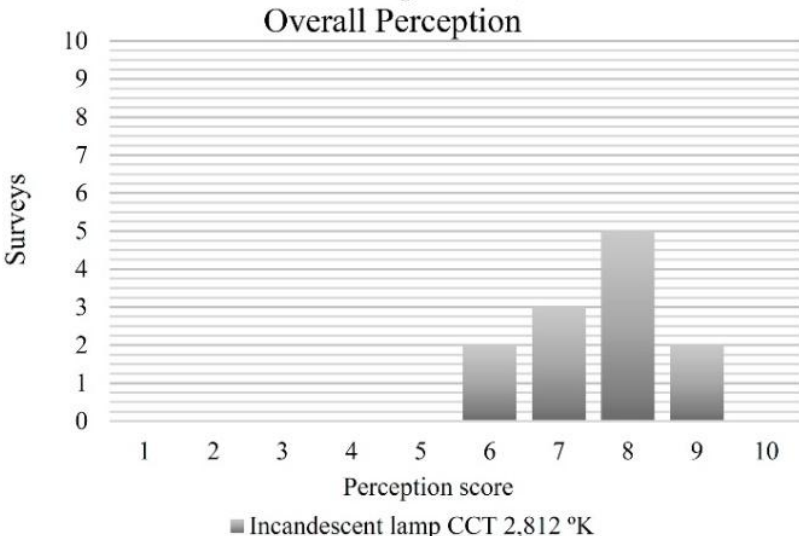

Fig. 11. Overall color rendering perception for an incandescent lamp with CCT $2,812^{\circ} \mathrm{K}$

The analysis of the overall perception of the incandescent lamp by the 12 specialists is shown in Fig. 11.

As seen in Fig. 10 and Fig. 11, the color rendering perceived by the 12 specialists is better for the LED lamp, despite the fact the Color Rendering Index and the Color Quality Scale describe the incandescent lamp as a better light source to render the colors. Therefore, the color rendering is not properly represented by the current metrics.

Regarding the accuracy of the Daylight Spectrum Index, it can be deduced that the overall color rendering of a light source is better defined by this metric, showing a DSI of
$84.20 \%$ for the LED lamp and a DSI of $75.80 \%$ for the incandescent lamp.

The trial has been extended to the analysis of the color rendering of the different hues of the visible spectrum. In the case of blue hues, the color rendering is improved for the LED lamp, as can be deduced from the comparison of Fig. 12 and Fig. 13.

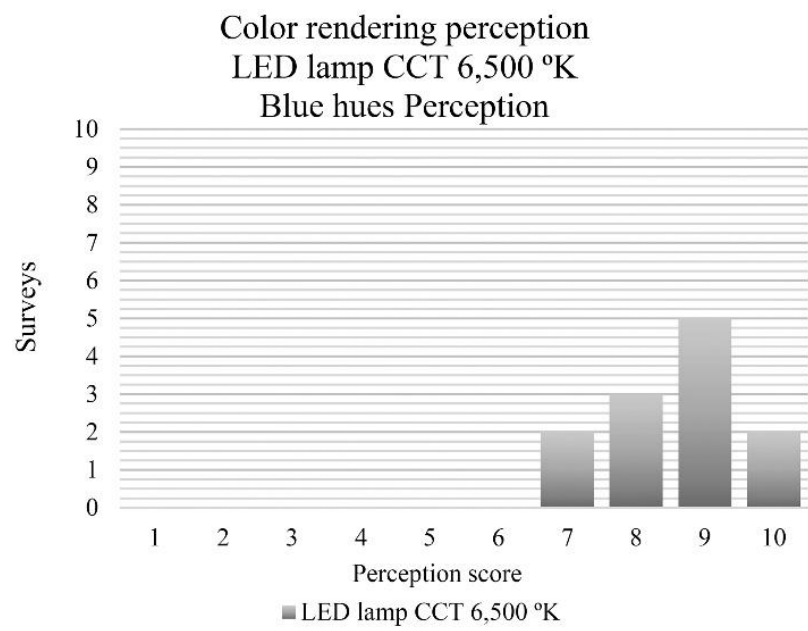

Fig. 12. Color rendering of the blue hues perception for a LED lamp with CCT $6,500^{\circ} \mathrm{K}$

Color rendering perception

Incandescent lamp CCT $2,812^{\circ} \mathrm{K}$

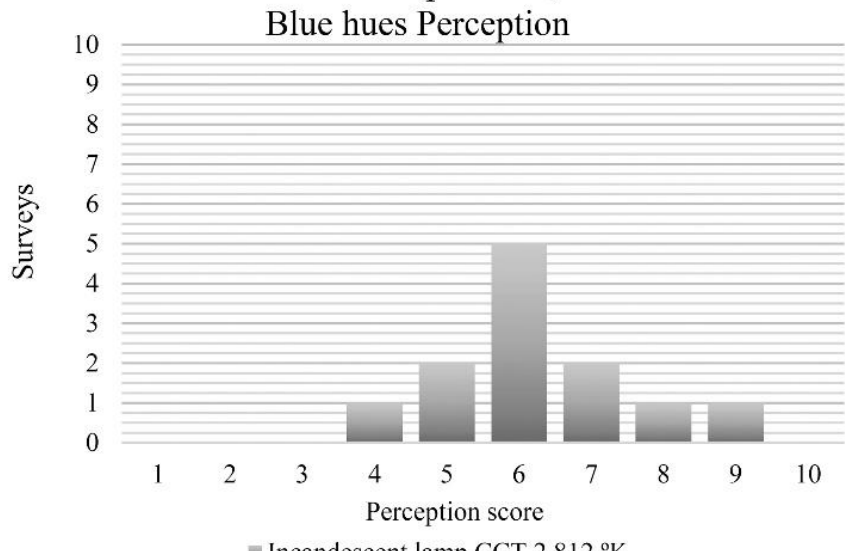

- Incandescent lamp CCT $2,812^{\circ} \mathrm{K}$

Fig. 13. Color rendering of the blue hues perception for an incandescent lamp with CCT $2,812^{\circ} \mathrm{K}$

Color rendering perception

LED lamp CCT $6,500^{\circ} \mathrm{K}$

Red hues Perception

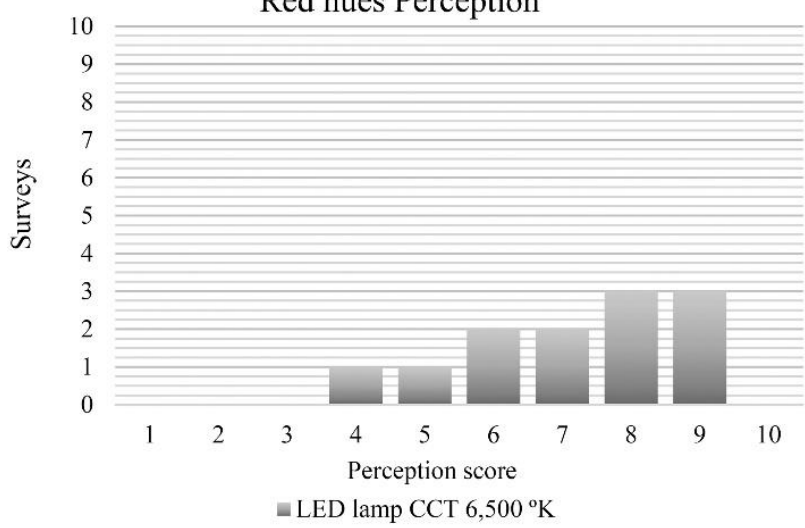

Fig. 14. Color rendering of the red hues perception for a LED lamp with CCT $6,500^{\circ} \mathrm{K}$ 
Color rendering perception

Incandescent lamp CCT $2,812^{\circ} \mathrm{K}$

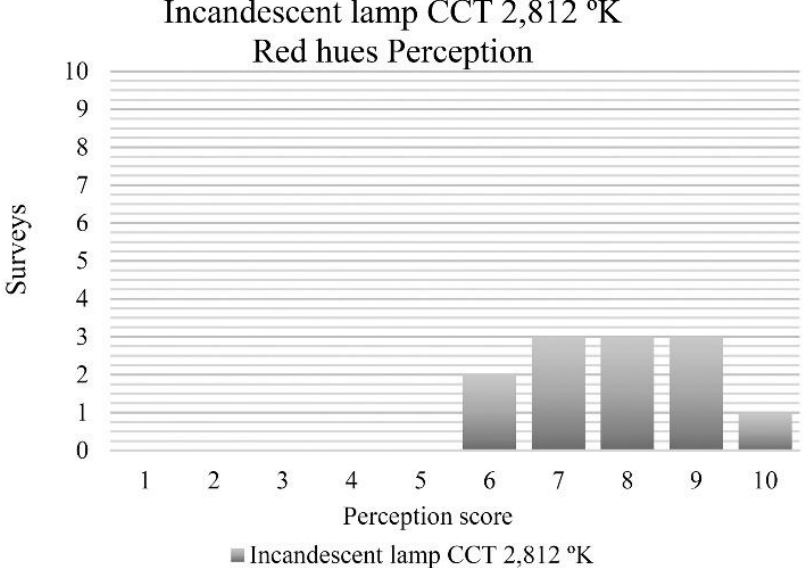

Fig. 15. Color rendering of the red hues perception for an incandescent lamp with CCT $2,812^{\circ} \mathrm{K}$.

As seen in previous Figures, the blue hues perception for the LED lamp corresponds to $85.80 \%$, whereas the incandescent lamp shows an average value of $62.50 \%$. Therefore, it can be deduced that the DSI better represents the color rendering than other metrics such as the CRI and CQS.

Following the methodology of this trial, the color rendering of the red hues is shown in Fig. 14 and Fig.15 respectively to LED and incandescent lamps.

As deduced from the previous Figures, the performance of the incandescent lamp is better than in the case of the LED lamp to render the red hues. The perception for this wavelength interval for the LED lamp corresponds to $71.60 \%$, whereas the incandescent lamp shows an average value of $78.30 \%$. Therefore, as in the case above, the DSI better defines the color rendering than previous metrics.

\section{CONCLUSIONS}

- The DSI allows an easy calculation of the color rendering of different light sources.

- The DSI does not depend on color temperature, so it can be used for any type of light source.

- The DSI can determine three main hue color renderings (blue, green and red), providing a better definition of the light source color performance.

- The DSI can be adapted according to the predominant sky conditions, so this metric can be adjusted to the location.

\section{REFERENCES}

[1] Commission Internationale de l'Eclairage. Method of measuring and specifying colour rendering properties of light sources. CIE Technical Report 13.3. Vienna: CIE, 1995.

[2] X. Guo and K. W. W, "Houser A review of colour rendering indices and their application to commercial light sources," Lighting Research and Technology, 2004, vol. 36, pp. 183-199.

[3] J. Schanda, "Colour rendering of light sources," Understanding the CIE System, New York: John Wiley, 2007, pp. 207-215.

[4] M. S. Rea and J. P. Freyssinier, "Color rendering: Beyond pride and prejudice," Color Research and Application, 2010.

[5] D. L. MacAdam, "Visual sensitivities to color differences in daylight," Journal of the Optical Society of America, 1942.

[6] R. Dangol, P. Bhusal, and L. Halonen, "Performance of colour fidelity metrics," Lighting Research and Technology, 2015, vol. 47, pp. 897-908.

[7] Commission Internationale de l'Eclairage. Colour rendering of white LED light sources. CIE Technical Report 177. Vienna: CIE, 2007.

[8] Commission Internationale de l'Eclairage. Colour rendering (TC1-33 closing remarks). CIE Technical Report 135. Vienna: CIE, 1999.

[9] W. Davis and Y. Ohno, "Color quality scale," Optical Engineering, 2010, vol. 49, pp. 1-16.

[10] W. Davis and Y. Ohno, "Toward an improved color rendering metric," in Proc. Fifth International Conference on Solid State Lighting, San Diego, California, USA, 2005.

[11] S. KAG, J. Schanda, L. Whitehead, and R. M. Luo, "CRI2012: A proposal for updating the CIE colour rendering index," Lighting Research and Technology, 2013, vol. 45, pp. 689-709.

[12] C. Li, R. M. Luo, C. Li, and G. Cui, "The CRICAM02UCS colour rendering index," Color Research and Application, 2012, vol. 37, pp. $160-167$.

[13] M. Royer and K. Houser, Understanding and Applying TM-30-15. Illuminating Engineering Society of North America, 2015.

[14] Commission Internationale de l'Eclairage. CIE Colorimetry - Part 2. Standard Illuminants for Colorimetry, CIE S 014-2/E:2006.

[15] Commission Internationale de l'Eclairage. CIE $19882^{\circ}$ Spectral Luminous Efficiency Function for Photopic Vision. CIE 086-1990.

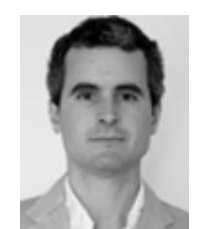

I. Acosta is a professor at the Department of Building Construction, University of Seville, Spain. He is member of the research group TEP-130 which is focused on sustainability, energy efficiency, lighting and acoustics related to building design.

The author belongs to the Instituto Universitario de Arquitectura y Ciencias de la Construcción.

The main research field of the author is the study of daylighting and its relationship with the architectural design. Most of the papers developed by the author analyze the energy savings in electric lighting produce by the proper use of daylighting, the study of predictive methods to determine the illuminance in courtyards and the measurement of the circadian stimulus. 